Rev. Psicol. (Arequipa. Univ. Catól. San Pablo) / Año 2020 / Vol 10 / N 1 / pp. 23-37

ISSN 2306-0565 versión impresa / ISSN 2311-7397 versión on line

(c) $(\$)$ Esta obra está bajo una Licencia Creative Commons

Atribución 4.o Internacional (BY-NC-ND)

\title{
RESILIENCIA Y APOYO SOCIAL PERCIBIDO \\ EN ESTUDIANTES DE 4TO Y 5TO \\ DE SECUNDARIA DEL DISTRITO DE AYAVIRI
}

\author{
RESILIENCE AND PERCEIVED SOCIAL SUPPORT AMONG \\ 4TH AND 5TH GRADE HIGH SCHOOL STUDENTS IN AYAVIRI
}

\author{
María Daniela Canazas Bustíos, Luz Marina Díaz Huayna \\ y Gabriela Ladid Cáceres Luna \\ Universidad Católica San Pablo, Arequipa, Perú
}

\begin{abstract}
Resumen
Según investigaciones realizadas en Ayaviri, los adolescentes presentan niveles altos de analfabetismo, deserción y problemas de conducta; además, el 12\% de embarazos corresponde a adolescentes y es una cifra que va en aumento. Es así que se evidencia la vulnerabilidad de la muestra evaluada, por lo que el presente estudio plantea determinar la relación entre los niveles de resiliencia y apoyo social percibido que les permitan afrontar estas circunstancias. Se asumió un diseño no experimental, transversal y correlacional. La muestra estuvo compuesta por 282 estudiantes de cuarto y quinto de secundaria del distrito de Ayaviri, Puno y las variables consideradas fueron edad, sexo y tipo de institución educativa a la que pertenecen. Los instrumentos empleados fueron la Escala de Resiliencia de Wagnild y Young, y Escala Multidimensional de Apoyo Social Percibido. En los resultados se obtuvo que existe una relación positiva entre ambas variables de estudio, la cual no parece estar influenciada por las variables demográficas, siendo el apoyo social percibido por parte de otras personas significativas el que mostró mayor respaldo estadístico. Asimismo, se pudo concluir que los evaluados poseen un nivel medio de apoyo social y de resiliencia. Estos resultados sirven para implementar programas de intervención y prevención a fin de reforzar los lazos entre la comunidad, mejorar el desarrollo y afianzar las capacidades de afrontamiento ante situaciones adversas y disminuir las conductas de riesgo.
\end{abstract}


Palabras clave: Resiliencia, apoyo social percibido, adolescencia.

\begin{abstract}
Research done in Ayaviri indicates that adolescents there have high levels of illiteracy, truancy and behavioral problems; in addition, 12\% of pregnancies occur among adolescents and this rate is increasing. Thus, the vulnerability of the evaluated sample is evident; hence, the present study proposed to determine the relationship between the levels of resilience and perceived social support that allow them to face these circumstances. A non-experimental, transversal and correlational design was used. The sample was composed of 282 Ayaviri, district of Puno, fourth and fifth grade high school students. The demographic variables considered were age, sex and the type of educational institution students attended. The scales used to measure these two variables were: the Resilience Scale by Wagnild \& Young and the Multidimensional Scale of Perceived Social Support. The results showed a positive relationship between the results obtained on the two scales. The results did not seem to be influenced by demographic variables. The variable "Social Support Perceived by Other Significant People" demonstrated the highest impact. The study also evidenced that the participating students had an average level of perceived social support and resilience. These results suggest the need to implement intervention and prevention programs aimed to strengthen ties within the community, to improve personal development of its adolescents, and to encourage them to develop additional skills helpful in coping with adverse situations and in reducing risky behaviors.
\end{abstract}

Key words: Resilience, perceived social support, adolescence.

\section{Introducción}

La adolescencia es una etapa que abarca desde los once hasta los veinte años, en la cual la persona logra la madurez biológica y sexual y busca alcanzar la madurez emocional y social (Feldman, Olds \& Papalia, 2001). Piaget (2001) menciona que los adolescentes experimentan distintas situaciones en las que cumplen diversos roles y experimentan cambios físicos, cognitivos y psicológicos que van a contribuir a que vayan construyendo su propia identidad. En este periodo del desarrollo, como lo indica Maddaleno (1994, citado por Del Águila \& Prado, 2003), existen más posibilidades de experimentar diversos cambios en el área personal e interpersonal, así como hay más probabilidad de que se presenten riesgos a corto, mediano y largo plazo, por lo que se los puede considerar como una población más vulnerable en comparación a los adultos al no poseer las mismas herramientas para superar las dificultades que les son propias a estos últimos por su madurez (Camarata \& Woodcock, 2006). Esta realidad se hace particular en los sectores socioeconómicos bajos, en donde, los adolescentes requieren asumir responsabilidades 
que no están acordes con las tareas y los deberes propios de su edad, por ejemplo, empiezan a laborar por la necesidad de apoyar con gastos familiares, mostrando bajo rendimiento académico hasta llegar a la deserción escolar (Dolto, 1990).

Ayaviri es un distrito de la provincia de Melgar en el departamento de Puno, el cual presentaba un nivel total de pobreza de $34.2 \%$ y de pobreza extrema de $6.4 \%$ en el 2018 (Centro Nacional de Planeamiento Estratégico [CEPLAN], 2018), superando el promedio de pobreza a nivel nacional de $20.5 \%$ reportado por el Instituto Nacional de Estadística e Informática (INEI) en el 2018 (El Comercio, 2019). El Ministerio de la Mujer y Poblaciones Vulnerables (MIMP) presentó un informe en el 2014 en el que refiere haber detectado algunas variables familiares como la poca comunicación y la falta de empatía y de autocontrol que dan lugar a consecuencias negativas sobre el rendimiento escolar de los hijos, ausentismo escolar (principalmente en mujeres), deserción, analfabetismo, problemas de conducta hasta embarazos adolescentes. Específicamente, en la provincia de Melgar, el índice de atraso escolar asciende a $11.35 \%$ y el de deserción es de 3.85\% (Estadística de la Calidad Educativa [ESCALE], 2017). Por su parte, García y Quispe (2018), en su estudio, profundizaron en el estilo de vida de las familias de los alumnos de últimos años de las escuelas de Ayaviri, evidenciando que los padres, en su mayoría, trabajan en actividades de agricultura y ganadería, perciben bajos ingresos económicos y viven en zonas alejadas del centro de la ciudad en donde se ubican las instituciones educativas, por lo que los adolescentes viven sin la supervisión de un adulto o apoderado la mayor parte de tiempo. Uno de los últimos proyectos desarrollados en esta zona fue el llamado "Yachay Wasi Llapanchis" o "La casa donde aprendemos todos" por su traducción del quechua, el cual fue realizado por la fundación América Solidaria (2016), quienes previamente detectaron una clara necesidad de fortalecer capacidades educativas, reforzar los lazos y motivar que los padres participen activamente en el proceso educativo de sus hijos. Además, las principales problemáticas encontradas en la población fueron: alcoholismo, elevados indicadores de maltrato intrafamiliary poco uso y aprovechamiento de los recursos educativos (América Solidaria, s.f.).

Es debido a lo anteriormente mencionado que Díaz y Morales (2011) destacan la importancia de desarrollar la resiliencia en los adolescentes pues les va a brindar factores protectores para que puedan sobrellevar todos los cambios mencionados previamente y es fundamental que se empiece a inculcar desde la etapa escolar (Aguiar \& Acle-Tomasini, 2012; Chávez, 2016; Núñez, 2014; Vera-Bachmann, 2015), ya que es un factor importante de respuesta ante las situaciones hostiles y que les permitirá desarrollar capacidades como la toma de decisiones, la participación y la igualdad, también les permitirá enfatizar sus fortalezas para superar las adversidades y generar estrategias de afrontamiento (Grotberg, 2006). 
La resiliencia, según Wagnild y Young (1993), es un factor que ayuda a afrontar las adversidades y da lugar a una reacción diferente hasta el punto de generar conductas positivas, adaptación al suceso y que se logre obtener beneficios de ello. Asimismo, González (2016) y Aléx et al. (2005) mencionan que es una capacidad dinámica que sigue pautas diferentes a cada edad y se evidencia a lo largo de la vida. Y en lo que respecta a la relación de esta variable con el aspecto socioeconómico, Del Águila y Prado realizaron una investigación en el 2003 en la que concluyen que aquellas personas pertenecientes a una clase social baja tienen mayor fortaleza y se sobreponen con mayor facilidad ante las circunstancias que se les presente.

Por su parte, Barra y Méndez (2008) y Cueto, Muñoz y Saldarriaga (2011) coinciden en que la presencia de la familia es influyente en la socialización de los hijos, su percepción de apoyo social, su adaptación y su bienestar psicológico. Cardoso y Verner (2008) apoyan esta idea mencionando que, para poder contrarrestar los posibles episodios de riesgo, se necesita de la combinación de los aspectos a nivel individual, social y familiar. A este aporte brindado por estos 3 ámbitos se le llama apoyo social, el cual ha sido reconocido como un elemento que fomenta el desarrollo de la resiliencia y desempeña un rol de protección frente a las situaciones estresantes (Martínez, Nava, Soria \& Vega, 2010). Respecto a esta variable, Durá y Garcés (1991) y Orcasita y Uribe (2010) mencionan que no basta únicamente la mera presencia de un grupo de personas, sino que se debe dar una interacción emocional y social pues se han evidenciado beneficios relacionados a la salud y modos de afrontamiento del estrés reduciendo el nivel de morbilidad y mortalidad (Broadhead et al., 1983; Barra, 2004). Del mismo modo, funciona como reductor del impacto de los eventos estresantes de la vida, ayuda también a ser más flexible cognitivamente y tener mejores formas de afrontar las dificultades que se presenten, reduce la ansiedad y genera un sentimiento de seguridad y bienestar (Barra, 2004; Cohen \& Wills, 1985; Durá \& Garcés, 1991; García, Martínez \& Maya, 2001). Sin embargo, su efecto es más significativo cuando las personas atraviesan altos niveles de estrés (García et al., 2001). Complementando esto, Cienfuegos y Palomar (2007) refieren que es un factor primordial que permitirá salir adelante y, en muchos casos, superar la pobreza conjuntamente con la intervención de instituciones o el gobierno centrándose en fortalecer las relaciones entre los integrantes de la comunidad y el fomento de la solidaridad entre ellos.

Diversos estudios destacan la importancia del apoyo familiar, de pares y de la comunidad en general (Bravo \& Fernández, 2003; Cava \& Musitu, 2003; Dahlem, Farley, Zimet \& Zimet, 1988). Sin embargo, en las investigaciones de Cienfuegos y Palomar (2007) y de Rodríguez (2010), determinaron que, en situaciones de pobreza, la fuente de apoyo principal sería la comunidad pues es un grupo heterogéneo amplio, y es visto como una estructura que brindará mayor apoyo. Sin embargo, lo fundamental es el tipo de relación con el apoyador, más no quién 
o quiénes cumplan este rol (Barra, 2004; Cienfuegos \& Palomar, 2007).

Respecto a la relación de ambas variables, se mencionan inicialmente las investigaciones de Polo (2009), De las Olas, García, Gómez y HombradosMendieta (2016) y Fernández-Zabala, Ramos-Díaz, Revuelta, RodríguezFernández y Ros (2016) quienes encontraron una relación positiva entre ambas en muestras con diferentes características. Específicamente en poblaciones de bajo nivel socioeconómico, Alderete y Cardozo (2009) refieren que aquellos adolescentes que estaban expuestos a circunstancias difíciles, tenían dificultad para adaptarse socialmente, y Encina, Leiva y Pineda (2013) complementan esto refiriendo que «el apoyo social percibido, tanto a nivel familiar como de las amistades, ha mostrado ser significativo en su relación con la resiliencia» (p. 119), siendo el apoyo familiar en esta población más transcendental que el brindado por los amigos, pero ambos servirían para explicar los niveles de resiliencia de la persona. En cambio, Cienfuegos y Palomar (2007) y Rodríguez (2010) detectaron que en poblaciones de bajos recursos, perciben mayor apoyo por parte de su comunidad pues con ellos entablan una fuerte relación de cercanía. Por su parte, Del Águila y Prado (2003) concluyeron que tener bajos recursos no determina la resiliencia; sin embargo, hicieron énfasis en que los jóvenes de clase baja poseen mayor desarrollo de su creatividad, lo cual los ayuda a solucionar de mejor forma sus problemas y seguir adelante en lugar de estancarse y lamentarse.

En consideración a lo expuesto anteriormente, realizar un estudio en este ámbito es de especial relevancia pues el desarrollo de los adolescentes, en este contexto en particular, amerita de la resiliencia y el apoyo social percibido como factores que ayudan a la reducción de riesgos conjuntamente con el soporte de la familia, amistades y otras personas significativas, pues son influyentes e importantes en dicho periodo (Cohen, 2004; Fergus \& Zimmerman, 2005). Además, la literatura e investigaciones respecto al afrontamiento de situaciones complejas y los medios que lo hacen posible aún son escasas, sobre todo en contextos socioeconómicos bajos y especialmente en esta zona, pues hay muy poca información publicada y accesible. Es en respuesta a la necesidad de abordar esta problemática que se realizó esta investigación que tuvo como objetivo el relacionar el nivel de resiliencia y el de apoyo social percibido en estudiantes de cuarto y quinto de secundaria del distrito de Ayaviri.

La importancia del presente trabajo radica en la posibilidad de brindar la información recaudada y que ésta sirva como base para generar intervenciones preventivas a favor de losalumnosysus familias, así como delos centros educativosy la comunidad;además, servirá para la implementación de talleres o programas de apoyo que faciliten la superación de retos o dificultades a lo largo de la vida ya que las decisiones que tomen en la adolescencia influenciarán su futuro (Grotberg, 1996). 


\section{Método}

Para el desarrollo de esta investigación se asumió un diseño no experimental, transversal y correlacional ya que se pretende conocer la relación que existe entre el apoyo social y la resiliencia sin buscar entre ellas una relación de causa-efecto (Hernández, Fernández, \& Baptista, 2006).

\section{Participantes}

La muestra estuvo conformada por 282 jóvenes, 170 mujeres $(60.3 \%)$ y 112 hombres (39.7\%) cuyas edades fluctúan entre los 14 y 19 años $(\mathrm{M}=15.38$; $\mathrm{DE}=$ 0.906) y se encuentran cursando cuarto o quinto de secundaria en dos colegios públicos $(83.7 \% ; \mathrm{N}=236)$ y un colegio parroquial $(16.3 \%$; $\mathrm{N}=46)$ del distrito de Ayaviri en el departamento de Puno. Para que los estudiantes sean incluidos en la muestra debieron ser alumnos regulares de cuarto y quinto de secundaria, pertenecer a un nivel socioeconómico bajo, que según los niveles proporcionados por la Asociación Peruana de Empresas de Investigación de Mercados (APEIM) en el 2018, se considera que la población evaluada se ubica en los niveles socioeconómicos Bajo inferior y Bajo superior; y que decidan voluntariamente participar del estudio. La muestra fue elegida haciendo uso de un muestreo no probabilístico (Hernández et al., 2006).

\section{Instrumentos}

Se utilizó la Escala de Resiliencia de Wagnild y Young (1993), la cual fue adaptada por Acevedo (2011). De acuerdo a los resultados del análisis estadístico de las evaluaciones aplicadas, el nivel de confiabilidad obtenido a través del alfa de Cronbach es de .824. La prueba está compuesta por 25 reactivos, empleando una escala tipo Likert del 1 al 7 y toma en cuenta 2 factores principales: la competencia personal y la aceptación de uno mismo y de la vida.

También se utilizó la Escala Multidimensional de Apoyo Social Percibido de Dahem, Farley, Zimet y Zimet (1988) adaptada por Barriga, Jofré, Mendoza y Mosqueda (2015). Según el análisis estadístico aplicado a las evaluaciones realizadas en este estudio, el nivel de confiabilidad obtenido con el alfa de Cronbach es de .856 . La prueba consta de 12 reactivos, y para las respuestas emplea una escala tipo Likert de 1 a 4, considerando tres fuentes principales de apoyo social percibido: familia, amigos o pares y otras personas significativas.

\section{Procedimientos}

La Oficina Diocesana de Educación Católica (ODEC) fue la entidad que autorizó la evaluación en las instituciones educativas; además, se informó a las autoridades de los tres colegios sobre el objetivo, confidencialidad, anonimato y voluntariedad de la participación y se procedió a entregar a los alumnos el asentimiento informado, la ficha sociodemográfica y las pruebas, explicándoles detalladamente la forma de llenado de cada uno de los documentos y absolviendo las dudas presentadas en el momento. 


\section{Análisis de datos}

El procesamiento de datos fue cuantitativo a través de estadística descriptiva e inferencial. Se evaluó la normalidad a través de un análisis con los estadísticos de asimetría curtosis y las pruebas de Kolmogorov-Smirnov y Shapiro-Wilk con el cual se determinó que la distribución de la muestra es normal; por lo tanto, se utilizaron pruebas estadísticas de orden paramétrico. Es debido a esto que se utilizó el coeficiente de correlación de Pearson haciendo uso del software estadístico SPSS en su versión 22.

\section{Resultados}

Respecto al apoyo social percibido, se observa que el nivel obtenido por la mayor parte de las personas es el medio con un porcentaje de $47.9 \%$ y el porcentaje de alto es de $44.3 \%$ tal como se detalla en la Tabla 1. Cabe resaltar que la sumatoria de los niveles Medio y Alto es de 92.2\% lo cual significa que más del 90\% de la muestra posee relaciones positivas con su entorno, confía y percibe ayuda de ellos pero esto no llega a un nivel alto de apoyo social percibido.

Tabla 1. Porcentaje de cada nivel de apoyo social percibido

\begin{tabular}{|lcc|}
\hline Nivel de Apoyo social Percibido & Frecuencia & Porcentaje \\
\hline Bajo & 22 & $7.8 \%$ \\
Medio & 135 & $47.9 \%$ \\
Alto & 125 & $44.3 \%$ \\
\hline Total & 282 & $100 \%$ \\
\hline
\end{tabular}

En cuanto a la resiliencia, el nivel predominante hallado en la muestra es el de moderado (42.6\%) tal como se especifica en la Tabla 2. Esto significa que la mayor parte de las personas tienen buena capacidad de resolución de problemas, afrontamiento de los obstáculos y pueden transformarlos en algo positivo. Sin embargo, no llegan a un nivel alto de resiliencia. También se puede observar que la cantidad de personas que poseen escasa resiliencia representa casi la cuarta parte de la población, lo cual es importante tener en cuenta para orientar la intervención futura.

Tabla 2. Porcentaje de cada nivel de resiliencia

\begin{tabular}{|lrc|}
\hline Nivel de Resiliencia & Frecuencia & Porcentaje \\
\hline Escasa & 63 & $22.3 \%$ \\
Moderada & 120 & $42.6 \%$ \\
Alta & 99 & $35.1 \%$ \\
\hline Total & 282 & $100 \%$ \\
\hline
\end{tabular}


Se evidencia una correlación positiva entre la resiliencia y el apoyo social percibido $(\mathrm{r}=0.148 ; \mathrm{p}<0.05)$. Específicamente se observa que las personas que perciben apoyo social de otras personas ajenas a su familia o amigos, poseen una mayor competencia personal $(\mathrm{r}=0.196 ; \mathrm{p}<0.01)$, aceptan su vida y a sí mismos $(\mathrm{r}=0.253$; $\mathrm{p}<0.01) \mathrm{y}$, en general, tienen una alta resiliencia $(\mathrm{r}=0.233 ; \mathrm{p}<0.01)$; lo cual quiere decir que hay una correlación positiva estadísticamente muy significativa entre esta dimensión del apoyo social percibido con la resiliencia y sus dos dimensiones. Por otro lado, una persona que percibe un mayor apoyo social general tiene una mayor aceptación de sí mismo y de su vida $(\mathrm{r}=0.155 ; \mathrm{p}<0.01)$. Este grado de significancia de apoyo social percibido baja en aquellas personas con una alta competencia personal $(\mathrm{r}=0.127 ; \mathrm{p}<0.05)$ o con una alta resiliencia $(\mathrm{p}=0.148 ; \mathrm{p}<$ o.05) sin que esta correlación deje de ser estadísticamente significativa (Tabla 3 ).

Tabla 3. Correlación de Apoyo social percibido/resiliencia

\begin{tabular}{|lccc|}
\hline & $\begin{array}{c}\text { Competencia } \\
\text { personal }\end{array}$ & $\begin{array}{c}\text { Aceptación de uno } \\
\text { mismoy la vida }\end{array}$ & $\begin{array}{c}\text { Escala de } \\
\text { resiliencia }\end{array}$ \\
\hline $\begin{array}{l}\text { Apoyo social percibido } \\
\text { de pares }\end{array}$ & 0.055 & 0.040 & 0.054 \\
$\begin{array}{l}\text { Apoyo social percibido } \\
\text { de familia }\end{array}$ & 0.035 & 0.060 & 0.047 \\
$\begin{array}{l}\text { Apoyo social percibido } \\
\text { de otra persona } \\
\text { Escala de apoyo social } \\
\text { percibido }\end{array}$ & $0.196^{* *}$ & $0.253^{* *}$ & $0.233^{* *}$ \\
\hline
\end{tabular}

** La correlación es significativa en el nivel o,o1 (2 colas) $\mathbf{p}<\mathbf{0 . 0 1}$.

${ }^{*}$ La correlación es significativa en el nivel o,05 ( 2 colas) $\mathbf{p}<0.05$.

\section{Discusión}

Los resultados de esta investigación confirman la existencia de una relación positiva entre las variables de estudio, respondiendo así a la pregunta de investigación planteada previamente.

El resultado obtenido concuerda con las investigaciones de Masten, Narayan, y Wright (2013) y Omar (2008), quienes encontraron que el apoyo social se relaciona positivamente con la resiliencia, sirviendo de soporte para las situaciones difíciles. Así también, otros investigadores resaltan el apoyo familiar en relación con la resiliencia, la cual genera el desarrollo de la autoestima y de la habilidad de resolución de problemas (Castillo, 2013; Florenzano, 1997; Krauskopf, 2007; Masten et al., 2013; Muñoz, 2012). Además, se indica que el apoyo social percibido funciona como amortiguador y protector de las consecuencias de problemas al igual que la resiliencia, pero se requiere del apoyo externo (Barra, 
2004), por tanto, ambas variables son relevantes para que el individuo supere y encuentre respuestas adaptativas para poder enfrentarse a las adversidades de la vida, siendo de vital importancia contar con una red de apoyo estable de amigos, familiares $y$ otras personas significativas.

En los resultados se obtuvo que la correlación más fuerte se dio entre las dimensiones de resiliencia y otras personas significativas, lo cual concuerda con las investigaciones revisadas en las que trabajaron con muestras similares a la empleada. En este estudio se refiere que las personas de bajo nivel socioeconómico buscan apoyo social en su comunidad ya que encuentran mayores posibilidades de salir adelante (Cienfuegos \& Palomar, 2007; Rodríguez, 2010). Asimismo, Gracia y Herrero (2006), encontraron en su investigación que en la comunidad se percibe un sentimiento de pertenencia e integración principalmente en poblaciones pequeñas donde todos se conocen, es así como se ve aumentado el nivel de autoestima de los integrantes de dicha comunidad. Por otro lado, Encina et al. (2013) y Fernández-Zabala et al. (2016) obtuvieron resultados diferentes, pues ellos destacan el apoyo de los amigos en esta etapa del desarrollo, y refieren que es más trascendental que el de la familia o que de la misma comunidad, ya que sería el que predice la satisfacción positiva.

El nivel más elevado obtenido por la mayor parte de los estudiantes en la prueba de apoyo social es el de medio seguido del alto, y es marcada la diferencia con la cantidad de alumnos que obtuvieron un puntaje bajo en esta escala; lo cual podría estar determinado por el entorno social en el que habitan ya que se evidencia una buena convivencia entre las personas de la comunidad lo que parece garantizar el establecimiento de vínculos positivos entre ellos.

Por otra parte, en cuanto a la resiliencia, los niveles con mayor representatividad de la muestra son los de moderado alto; sin embargo, la diferencia con el nivel escaso no es tan marcada. Esto podría explicarse porque los alumnos se encuentran en la adolescencia y, al ser esta una etapa de transición entre la niñez y la adultez, están en proceso de formar su identidad y tomar sus propias decisiones (Fergusson \& Lynskey, 1996), pero a su vez, muestran una mayor inestabilidad, la cual puede afectar su capacidad de afrontamiento ante las situaciones adversas que se les presenten.

En lo que respecta a tipo de escolaridad, en la población en la que se efectuó el estudio predominan las escuelas públicas, por lo que no se pueden generalizar los resultados de este análisis. Un dato a tomar en cuenta es que, al parecer, la educación brindada en las escuelas parroquiales es más integral y apoyan a los alumnos a desarrollar su propia identidad y así poder tomar mejores decisiones para afrontar todo lo que se les presente pues desde pequeños les brindan una educación de calidad, conjuntamente con una formación moral basada en valores y fomentando el apoyo familiar (Cortez, 2007). 
El relacionar las variables mencionadas ayuda a entender e identificar la importancia que tienen en el desarrollo de la persona, pues proporciona mayor información y distintas alternativas para poder implementar programas de intervención y prevención para la población evaluada y sus familias los cuales pueden estar orientados a reforzar los lazos entre la comunidad y trabajar en la superación personal a fin de que los miembros puedan acceder a recursos de apoyo, estabilidad y seguridad. Por las características descritas de la muestra, son adolescentes de bajos recursos y algunos de ellos ya trabajan, entonces, el proporcionarles herramientas para afrontar las situaciones difíciles, podrán superarse, pues según Del Águila y Prado (2003), aquellas personas de bajo nivel socioeconómico buscarían diferentes elementos para poder solucionar sus problemas de la mejor manera.
Los resultados de esta investigación también brindan información relevante que ayuda a describir una población de la cual se cuenta con pocas investigaciones previas y puede dar pie a estudios futuros que permitan conocer si es que hay otros factores que influyan en las variables de estudio como la estructura familiar, estilos de crianza o las mismas características de la localidad en la que habitan. Sería recomendable también considerar el uso de una prueba que brinde resultados más específicos y permita determinar la fuente principal de apoyo social percibido a fin de profundizar el análisis. Asimismo, se podría replicar la investigación en centros educativos públicos, privados y parroquiales a fin de contrastar los resultados obtenidos; teniendo en cuenta el empleo de muestras homogéneas según cada variable considerada. 


\section{Referencias}

Acevedo, A. (2011). Bienestar psicológico y resiliencia en pacientes oncológicos con tratamiento de quimioterapia del hospital Belén de Trujillo. (Tesis de pregrado). Universidad César Vallejo, Trujillo, Perú.

Aguiar, E., \& Acle-Tomasini, G. (2012). Resiliencia, factores de riesgo y protección en adolescentes mayas de Yucatán: elementos para favorecer la adaptación escolar. Acta Colombiana de Psicología, 15(2), 53-64.

Alderete, A., \& Cardozo, G. (2009). Adolescentes en riesgo psicosocial y resiliencia. Psicología desde el Caribe, 23, 148-182.

Aléx, L., Gustafson, A., Jonsén, E., Lundman, B. Norberg, A., \& Nygren, B. (2005). Resilience, sense of coherence, purpose in life and self-transcendence in relation to perceived physical and mental health among theoldest old. Aging E Mental Health, 9(4), 354-362. doi: $10.1080 / 1360500114415$

América Solidaria (2016). Ayaviri: creciendo y aprendiendo en comunidad. Recuperado de: http://americasolidaria.org/informate/ayaviri-peru-cierre-primer-ciclo-proyecto/

América Solidaria (s.f.). Fortaleciendo capacidades educacionales en Ayaviri. Recuperado de: http://americasolidaria.org/proyectos/fortaleciendo-capacidades-educacionales/

Asociación Peruana de Empresas de Investigación de Mercados (2018). Niveles socioeconómicos 2018. Recuperado de: http://www.apeim.com.pe/wp-content/themes/apeim/ docs/nse/APEIM-NSE-2018.pdf

Barra, E. (2004). Apoyo social, estrés y salud. Psicología y Salud, 14(2), 237-243.

Barra, E., \& Méndez, P. (2008). Apoyo social percibido en adolescentes infractores de ley y no infractores. Psykhe, 7(1), 59-64. doi: 10.4067/So718-222820080oo10ooo6

Barriga, O., Jofré, V., Mendoza, S., \& Mosqueda, A. (2015). Validez y confiabilidad de una escala de apoyo social percibido en población adolescente. Enfermería Global, 14(39), 125-136. doi: 10.6018/eglobal.14.3.200551

Bravo, A., \& Fernández, J. (2003). Las redes de apoyo social de los adolescentes acogidos en residencias de protección. Un análisis comparativo con población normativa. Psicothema, 15(1), 136-142. 
Broadhead, W., Crimson, R., Gehlbach, S., Heyden, S., James, S., Kaplan, B.,...Wagner, E. (1983). The epidemiologic evidence for a relationship between social supportand health. American Journal of Epidemiology, 117 (5), 521-537. doi:10.1093/oxfordjournals.aje.a113575

Camarata, S., \& Woodcock, R. (2006). Sex differences in processing speed: Developmental effects in males and females. Intelligence, 34(3), 231-252. doi: 10.1016/j.intell.2005.12.001

Cardoso, A., \& Verner D. (2008). Youth risk-taking behavior in Brazil: drug use and teenage pregnancy. Institute for the Study of Labor, Discussion Paper Series, 3030(2-14). doi: $10.1596 / 1813-9450-4548$

Castillo, E. (2013). Resiliencia, percepción de apoyo social, apoyo familiar y autoestima, en estudiantes de establecimientos municipales con alto índice de vulnerabilidad escolar, de la comuna de Talca. (Tesis de pregrado). Universidad de Talca, Chile.

Cava, M., \& Musitu, G. (2003). El rol del apoyo social en el ajuste de los adolescentes. Intervención Psicosocial, 12(2), 179-192.

Centro Nacional de Planeamiento Estratégico (2018). Información departamental, distrital y provincial que requiere atención adicional y devengado per cápita. Recuperado de: https://www.ceplan.gob.pe/wp-content/uploads/2018/o9/Matriz-de-indicadores-al30-de-junio-2018-VF-1.pdf

Chávez, E. (2016). Fomento de la resiliencia contra la deserción en la educación superior (Documento de trabajo). Universidad Autónoma de Zacatecas, México.

Cienfuegos, Y., \& Palomar, J. (2007). Pobreza y apoyo social: Un estudio comparativo en tres niveles socioeconómicos. Interamerican Journal of Psycohology, 41(2), 177-188.

Cohen, S. (2004). Social relationships and health. American Psychologist, 59(8), 676-684. doi: 10.1037/0003-066X.59.8.676.

Cohen, S., \& Wills, T. (1985). Stress, social support, and the buffering hypothesis. Psychological Bulletin, 98(2), 310-357. doi: 10.1037/0033-2909.98.2.310

Cortez, C. (2007). Presencia de las escuelas y los colegios parroquiales católicos en la educación peruana. Studium Veritatis, 6(10-11), 193-238.

Cueto, S., Muñoz, I., \& Saldarriaga, V. (2011). Conductas de Riesgo entre adolescentes peruanos: un enfoque longitudinal. En Salud, interculturalidad y comportamiento de riesgo (pp. 119-165). Lima, Perú: GRADE. 
Dahlem, N., Farley, G., Zimet, G., \& Zimet, S. (1988). The multidimenional scale of perceived social support. Journal of personality assessment, 52(1), 30-41. doi: 10.1207/ S15327752jpa5201_2

Delas Olas, M., García, A., Gómez, L., \& Hombrados-Medieta, I. (2016). Resiliencia, apoyo social y satisfacción vital de la población inmigrante. En D. Carbonero, N. Caparros, C. Gimeno \& E. Raya (Coords.), Respuestas transdisciplinarias en una sociedad global. Aportaciones desde el Trabajo Social (pp. 1-11). Logroño, España: Universidad dela Rioja.

Del Águila, M., \& Prado, R. (2003). Diferencia en la resiliencia según géneroy nivel socioeconómico en losadolescentes. Persona, 6, 179-196. doi:10.26439/persona2003.noo6.885

Díaz, D., \& Morales, M. (2011). Estudio comparativo de la resiliencia en adolescentes: el papel del género, la escolaridad y procedencia. Uaricha Revista de Psicología (Nueva época), 8(17), 62-77. Recuperadodehttps://docplayer.es/18802110-Estudio-comparativo-de-la-resiliencia-en-adolescentes-el-papel-del-genero-la-escolaridad-y-procedencia.html

Dolto, F. (1990). La causa de los adolescentes. Barcelona, España: Seix Barral.

Durá, E., \& Garcés, J. (1991). La teoría del apoyo social y sus implicaciones para el ajuste psicosocial de los enfermos oncológicos. Revista de Psicología Social, 6(2), 257-271. doi: 10.108o/02134748.1991.10821649

El Comercio (2019). Pobreza monetaria y pobreza extrema en el Perú disminuyó en el 2018. Recuperado de: https://elcomercio.pe/economia/peru/ pobreza-monetaria-pobreza-extrema-peru-disminuyo-2018-noticia-625267

Encina, Y., Leiva, L., \& Pineda, M. (2013). Autoestima y apoyo social como predictores de la resiliencia en un grupo deadolescentes en vulnerabilidad social. Revista de Psicología, 22(2), 111-123. doi: 10.5354/o719-0581.2013.30859

Estadística de la Calidad Educativa (2017). Puno: ¿cómo vamos en educación? Recuperado de: http://escale.minedu.gob.pe/c/document_library/ get_file?uuid=7efbe7bf-5a51-4b6e-a289-5ce4bfac8d6a\&groupId=10156

Feldman, R., Olds, S., \& Papalia, D. (2001). Desarrollo humano. Bogotá: McGraw-Hill.

Fergus, S., \& Zimmerman, M. (2005). Adolescent resilience:A Framework for understanding health development in the face of risk. Annual Review of Public Health, 26(1), 399-419. doi: 10.1146/annurev.publhealth.26.021304.144357 
Fergusson, D. \& Lynskey, M. (1996). Adolescent resiliency to family adversity. Journal of Child Psychology and Psychiatry, and Allied Disciplines, 37(3), 281-292. doi:10.111//j.14697610.1996.tbo1405.x

Fernández-Zabala, A., Ramos-Díaz, E., Revuelta, L, Rodríguez-Fernández, A., \& Ros, I. (2016). Bienestar subjetivo en la adolescencia: el papel de la resiliencia, el autoconcepto y el apoyo social percibido. Suma Psicológica, 23(1), 6o-69. doi:10.1016/j.sumpsi.2016.02.002

Florenzano, R. (1997). El adolescentey sus conductas de riesgo. SantiagodeChile:Universidad Católica de Chile.

García, M., Martínez, M., \& Maya, I. (2001). El efecto amortiguador del apoyo social sobre la depresión en un colectivo de inmigrantes. Psicothema, 13(4), 605-610.

García, R., \& Quispe, M. (2018). Inteligencia emocional en adolescentes con familias integradas y desintegradas de instituciones educativas secundarias del distrito de Ayaviri - Puno, 2018. (Tesis de Pregrado). Universidad Nacional deSan Agustín, Arequipa, Perú.

González, N. (2016). Resiliencia y Personalidad en niños y adolescentes. Cómo desarrollarse en tiempos de crisis. México: Ediciones Eón.

Gracía, E., \& Herrero, J. (2006). La comunidad como fuente de apoyo social: evaluación e implicaciones en los ámbitos individual y comunitario. Revista Latinoamericana de Psicología, 38(2), 327-342.

Grotberg, E. (1996). Guía de promoción de la resiliencia en los niños para fortalecer el espíritu humano. Holanda: Bernard Van Leer.

Grotberg, E. (2006). La resiliencia en el mundo de hoy. Cómo superar las adversidades. España: Gedisa.

Hernández, R., Fernández, C., \& Baptista, P. (2006). Metodología de la investigación. México: McGraw-Hill.

Krauskopf, D. (2007). Sociedad, adolescencia y resiliencia en el siglo XXI. En D. Krauskopf, M. Munist, T. Silver \& E. Suárez (Comps.), Adolescencia y Resiliencia (pp. 19-36). Buenos Aires: Paidós.

Martínez, L., Nava, C., Soria, R., \& Vega, C. (2010). Calidad de red como variable moduladora de depresión en adolescentes estudiantes de nivel medio. Liberabit, 16(1), 105-112. 
Masten, A., Narayan, A., \& Wright, M. (2013). Resilience processes in development: four waves of research on positive adaptation in the context of adversity. En S. Goldstein \& R. Brooks (Eds.), Handbook of resilience in children (pp. 15-37). Nueva York, Estados Unidos: Springer.

Muñoz, P. (2012). Influencia de los factores comunitarios en la resiliencia: estudio realizado con hogares urbanos de la zona central de la república mexicana, beneficiarios del Programa de Desarrollo Humano Oportunidades. (Tesis doctoral). Universidad Iberoamericana, México.

Núñez, D. (2014). Capacidades resilientes en adolescentes en contexto de vulnerabilidad. (Tesis de pregrado). Universidad Academia de Humanismo Cristiano, Chile.

Omar, A. (2008). Bienestar subjetivoy perspectivas de futuro como predictores de resiliencia en adolescentes. En J. Moysén (Ed.), El claroscuro de la vida juvenil (pp. 5-22). México: Editorial de la Universidad Juárez del Estado de Durango.

Orcasita, L., \& Uribe, A. (2010). La importancia del apoyo social en el bienestar de losadolescentes. Psychologia: Avances de la Disciplina, 4(2), 69-82. doi: 10.21500/19002386.1151

Piaget, J. (2001). La representación del mundo en el niño. Madrid, España: Morata.

Polo, C. (2009). Resiliencia: Factores protectores en Adolescentes de 14 a 16 años. (Tesis de pregrado). Universidad del Aconcagua, Chile.

Rodríguez, S. (2010). Relación entreel nivel socioeconómico, apoyo social percibido, género y depresión en niños. Interdisciplinaria. Revista de Psicología y Ciencias Afines, 27(2), 261-275.

Vera-Bachmann, D. (2015). Resiliencia, pobrezay ruralidad [Carta al editor]. Revista Médica de Chile, 143(5), 677-678. doi: 10.4067/Soo34-98872015000500018

Wagnild, G., \& Young, H. (1993). Developmentand psychometric evaluation of the Resilience Scale. Journal of Nursing Measurement, 1(2), 165-178.

Recibido: 15 de noviembre de 2019

Aceptado: 5 de marzo de 2020 